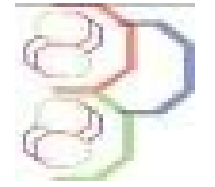

Journal of Applied Biosciences 145: 14914 - 14929

\title{
Impacts socio-environnementaux des activités agricoles en zone de montagnes au Nord-Ouest de l'Atacora au Bénin
}

\author{
Pocoun Damè Kombienou183 *; Ismaël Imorou Toko283 ; Gustave Dieudonné Dagbenonbakin1 ; Guy \\ Apollinaire Mensah ${ }^{1} \&$ Brice Augustin Sinsin ${ }^{3}$ \\ 1 Institut National des Recherches Agricoles du Bénin (INRAB); 01BP 884 Cotonou, Bénin. \\ 2 Laboratoire de Cartographie (LaCarto), Institut de Géographie, de l'Aménagement du Territoire et de \\ l'Environnement, Université d'Abomey-Calavi; 01BP 526 Cotonou, Bénin. \\ 3 Laboratoire d'Écologie Appliquée de la FSA/UAC, 01BP 526 Cotonou, Bénin \\ $\left(1 \& 3^{*}\right)$ Adresse du principal Auteur: e-mail ykopoda@yahoo.fr.123* $;(229) 97355649 / 95454959$
}

Original submitted in on $18^{\text {th }}$ October 2019. Published online at www.m.elewa.org/journals/ on $31^{\text {st }}$ January 2020 https://doi.org/10.35759/JABs.145.7

\section{RESUME}

L'étude aborde la dynamique de l'utilisation des terres à travers l'évolution des emblavures et de la production des principales cultures, puis l'impact des activités agricoles sur l'environnement et les conditions de vie des populations.

Objectif: L'objectif global de l'étude était d'évaluer les impacts socio-environnementaux des activités agricoles dans les Communes de Natitingou, Boukombé, Tanguiéta et Toucountouna en zone de montagnes au nord-ouest de l'Atacora au Bénin.

Méthodologie et résultats: Les méthodes utilisées étaient les enquêtes exploratoires, les diagnostics participatifs, les sondages d'opinion et les analyses statistiques tels que la variance suivant le test de Tukey et le test de corrélation. Au total, 400 agriculteurs sont sélectionnés au hasard et interrogés dans huit villages des quatre communes à l'aide d'un questionnaire structuré pour obtenir des données relatives aux nombres de cultures produites sur une même parcelle, aux différentes pratiques agricoles, à l'utilisation des engrais minéraux, à l'adoption des techniques endogènes et exogènes de gestion et de conservation de la fertilité des sols dans le milieu. Les résultats ont montré que les systèmes de productions agricoles étaient encore de type extensif et itinérant sur brûlis. Les cultures étaient mises en place en pure et en associations entre avril et décembre avec une prééminence des céréales qui ont occupé environ $52 \%$ des superficies emblavées. Les productions étaient plus en fonction des superficies que de l'intensification agricole. Les causes de dégradation des sols, la production, ont été collectées lors des entretiens collectifs et individuels. Les impacts de la production agricole se traduisaient surtout entre autres, par la disparition du couvert végétal, la baisse de la fertilité des sols et la pollution de certains cours d'eau aux bords desquels se réalisaient des activités à dominance agricole.

Conclusion et application des résultats : Certaines activités telles que l'utilisation des engrais chimiques et les feux de végétation ont résolu d'une manière ponctuelle certains problèmes comme l'amélioration des rendements agricoles et l'augmentation des revenus, mais elles n'ont pas duré dans le temps. Au fil des années, ces activités ont eu un impact négatif sur le milieu et les conditions de vie des populations. L'intensification agricole basée sur la vulgarisation et l'adoption des technologies plus productives et 
respectueuses de l'environnement, efficientes, facilement applicables par les producteurs, demeure l'une des stratégies pour garantir une utilisation durable des ressources naturelles.

Mots clés: Bénin, Atacora, activités agricoles, impacts socio-environnementaux et dégradation des sols

\section{ABSTRACT}

The study approaches the dynamics of the land use through the evolution of cultivated area and the production of the principal crops, then the impacts of the agricultural activities on the environment and the living conditions of the population.

Objective: The total objective of the study was to evaluate the socio-environmental impacts of the agricultural activities in the Communes of Natitingou, Boukombe, Tanguieta and Toucountouna in mountainous area in the North-Western of Atacora in Benin.

Methodology and Results: The methods used were the exploratory investigations, the participative diagnoses, the opinion polls and statistical analyses such as the variance according to the test of Tukey and the test of correlation. On the whole, 400 farmers were selected randomly and questioned in eight villages of the four communes using a structured questionnaire to obtain relative data with the numbers of cultures produced on the same piece, with various husbandries, with the use of mineral manures, the adoption of the endogenous and exogenous technical of management and conservation of the fertility of the grounds in the medium. The results showed that agricultural production systems were still extensive and itinerant on slash-and-burn. Crops were established in pure and association between April and December with a pre-eminence of cereals, which accounted for about $52 \%$ of the area. Productions were more based on acreage than agricultural intensification. The causes of soil degradation, production, were collected during collective and individual interviews. The impacts of agricultural production were mainly reflected in the disappearance of vegetation cover, the decline in soil fertility and the pollution of certain rivers along which agricultural-dominated activities were carried out.

Conclusion and application of results: Some activities such as the use of chemical fertilizers and wildfires have solved problems such as improving agricultural yields and increasing incomes on an ad hoc basis, but they didn't last. Over the years, these activities have negatively affected the environment and living conditions of the population. The agricultural intensification based on the popularization and the adoption of more productive and environment friendly technologies, easily applicable by the producers, remains one of the strategies to guarantee a durable use of the natural resources.

Keywords: Benin, Atacora, agricultural activities, socio-environmental impacts and impoverishment of the soil

\section{INTRODUCTION}

L'un des problèmes auxquels les pays de l'Afrique de l'Ouest doivent apporter des solutions adéquates à moyen terme est la promotion de l'agriculture durable, c'est-à-dire respectueuse de l'environnement et prenant en compte le développement socio-économique de tous les acteurs impliqués. Alors que le taux de croissance démographique s'accélère dans la région, l'agriculture qui doit nourrir les populations et assurer leur mieux être connaît une crise caractérisée par une baisse relative de sa production, une dégradation des ressources naturelles et l'environnement. En effet, le développement rural mal maîtrisé, les pollutions diverses liées à l'usage incontrôlé des différents intrants agricoles, la baisse de la qualité des eaux, l'extension de l'érosion, l'appauvrissement des sols et le recul sans cesse du couvert forestier sont les manifestations les plus visibles de cette dégradation (Roufaï, 2005). Ainsi, les systèmes de productions agricoles pratiqués dans la zone montagneuse au nord-ouest de l'Atacora et les techniques culturales en cours ont un impact négatif sur le milieu naturel. Par ailleurs, les changements spatio-temporels de l'occupation des terroirs dus dans la plupart des cas aux systèmes de cultures ont des effets sur la fertilité des sols et par voie de conséquence les productions agricoles 
en Afrique. L'Afrique Sub-saharienne est confrontée à une croissance démographique importante avec des prévisions de doublement de sa population d'ici à 2050 (Dixon et al., 2001). Cette croissance est essentiellement urbaine et s'accompagne de besoins croissants en nourriture. Le Département de l'Atacora au nord-ouest du Bénin est astreint à ces contraintes. Ce secteur est souvent cité comme étant de grande pauvreté dans laquelle le problème de terres se pose avec acuité (Kiansi, 2011 ; Kombienou et al., 2016). Les populations de l'Atacora sont venues d'horizons divers pour des causes variées. Elles y sont installées malgré l'exiguïté de l'espace cultivable (Kombienou, 2010). La recherche s'articule autour de la dynamique des terroirs induits par les systèmes de production agricole et la fertilité des sols dans les agro-systèmes de montagne. Elle est menée dans les Communes de Natitingou, Boukombé, Tanguiéta et Toucountouna de la zone montagneuse au nord-ouest de l'Atacora de manière participative pour amener les populations

\section{MATERIEL ET METHODES}

Analyse des impacts environnementaux des activités agricoles au nord-ouest de l'Atacora

Outils de collecte des données: Les outils de collecte des données ci-après ont été utilisés : un questionnaire pour faire des enquêtes auprès des producteurs et autres acteurs intervenant dans le secteur agricole ; un guide d'entretien élaboré pour être utilisé en focus group ; une grille d'observation pour faire des observations.

Ces différents outils ont été complétés par des observations directes sur le terrain.

Techniques de collecte des données: Les techniques utilisées ont été les suivantes le diagnostic participatif au niveau de chaque village ;

$\checkmark$ les sondages d'opinions par tranches d'âges variant de 20-70 ans et supérieur à 70 ans.

$\checkmark$ les interviews structurées et semi-structurées ayant pour $\mathrm{N}=400$ agriculteurs (chefs d'exploitations) avec ni=50 agriculteurs par village (taille de l'échantillon dans l'ensemble des villages d'étude est 400). Le nombre 400 a représenté la base de sondage qui n'était rien d'autre que la liste des agriculteurs (chefs d'exploitations) ayant une partie de leurs exploitations sur les flancs de montagnes, âgés d'au moins 20 ans. Ce chiffre a été de 0,1 des producteurs de l'ensemble des villages de la zone d'étude, obtenu après à mettre en œuvre des pratiques d'exploitations durables de leurs ressources naturelles au niveau de leurs exploitations agricoles individuellement ou de façon collective. II est nécessaire d'évaluer les impacts socio-environnementaux des activités agricoles en zone montagneuse dans les quatre (04) Communes au nord-ouest de l'Atacora au Bénin afin de pallier cette situation. C'est dans l'optique de mieux apprécié l'état de l'environnement au nord-ouest de l'Atacora au Bénin que la recherche sur les «Impacts socioenvironnementaux des activités agricoles au nordouest de l'Atacora au Bénin» a été initiée. D'une manière spécifique, l'étude vise, à analyser l'état actuel des sols dans la zone d'étude, celui de la végétation et aptitudes culturales des sols. A moyen terme, les résultats de cette analyse serviront, de base à l'élaboration des stratégies de restauration des terres du nord-ouest de l'Atacora favorisant un accroissement de la production agricole.

épurement de la liste du recensement de la LEPI-2010 dans le Département de l'Atacora; les interviews individuelles autour des variables telles que activités agricoles, valeurs socio-environnementales des spéculations agricoles, perceptions paysannes des situations données auprès de différents groupes socioprofessionnels concernés. II a été aussi exploité les rapports du CeRPA-Atacora/Donga des années 1993 à 2013. Au total, les trois types d'entretiens suivants ont été conduits:

- Entretiens informels: Non structurés, ouverts, accordant une marge de manœuvre à l'enquête, ce type d'entretien a été surtout privilégié lors des enquêtes sur l'historique d'occupation des terres sur la chaîne, les données sur les groupes socioculturels et sur les relations qu'ils entretiennent, la description des pratiques et des processus, le recoupement des informations, la typologie selon le niveau de prospérité, etc. Ils ont été réalisés avec différents acteurs et représentants de groupes socioculturels rencontrés sur le terrain comme des personnes ressources, des producteurs, des ONG, des chefs traditionnels, des décideurs politiques locaux, etc.

- $\quad$ Entretiens semi-structurés : Les entretiens semi-structurels ont été réalisés avec un guide d'entretien conduit auprès de 400 exploitants agricoles. 
Les questions posées étaient à la fois ouvertes et fermées et concernaient surtout le niveau de diversité, les systèmes de culture, l'historique d'occupation des terres, la fréquence des paramètres et les déterminants socio-environnementaux.

- $\quad$ Entretiens de groupes: Plusieurs entretiens ont été conduits avec des petits groupes dans le village, afin de comprendre, au niveau local, les stratégies locales de gestion et de l'utilisation des terroirs. La figure 1 a synthétisé l'impact de la croissance démographique, des systèmes de production agricoles et de la variabilité saisonnière des pluies sur l'utilisation du terroir.

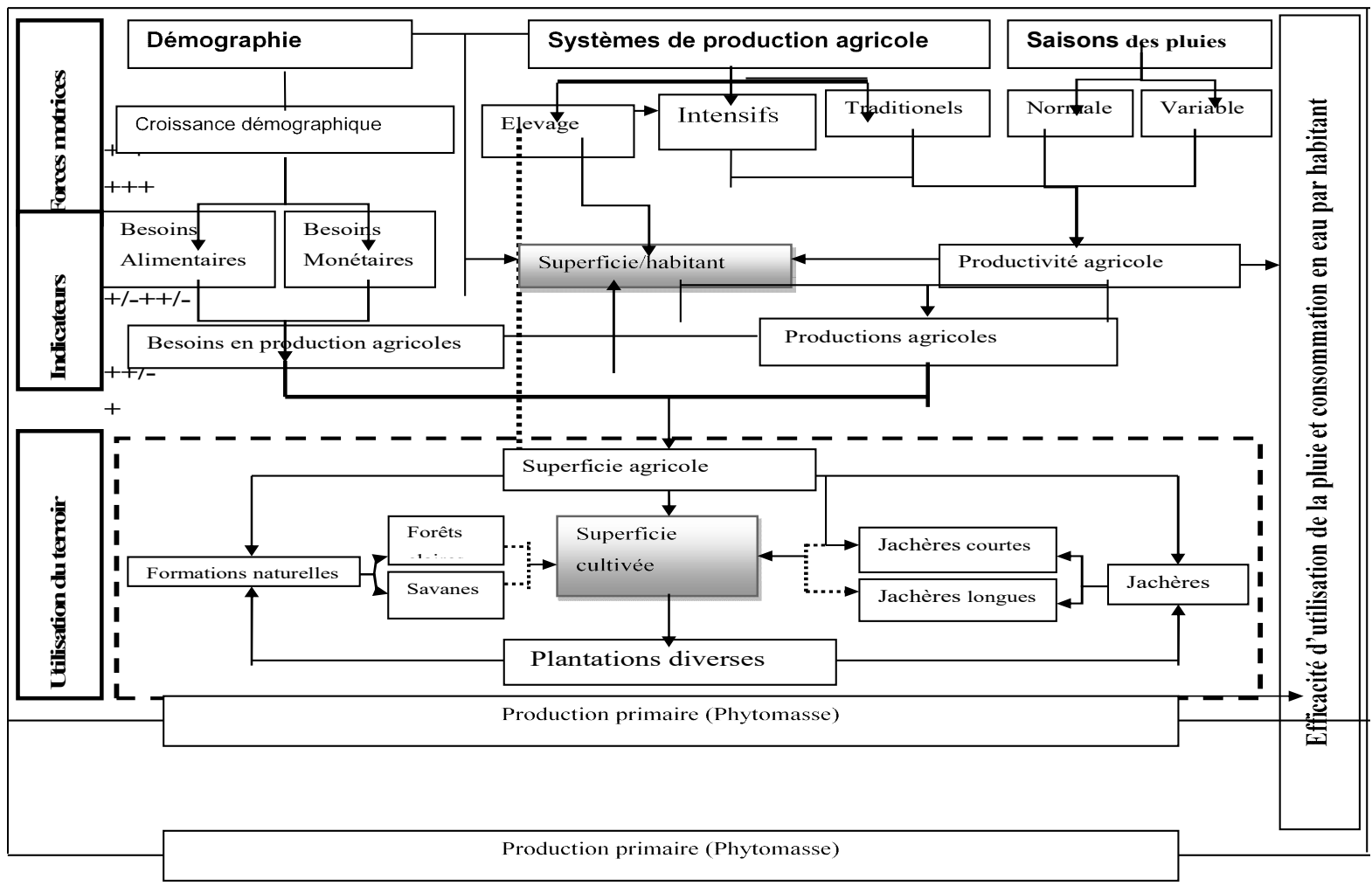

Figure 1 : Modèle d'impact de la croissance démographique, des systèmes de production agricoles et de la variabilité saisonnière des pluies sur l'utilisation du terroir

\section{Source : Travaux de terrain, 2011-2013}

Analyse et évaluation des impacts : La matrice de Léopold et al. (1971) et le cadre de référence de l'Agence Béninoise pour l'Environnement (ABE, 1999), ont servi à l'évaluation des impacts des activités agricoles sur les écosystèmes et les conditions de vie des populations rurales. La Matrice de Léopold et al. (1971) a permis de croiser les sources d'impacts (sous activités de l'agriculture) avec les composantes du milieu pour faire ressortir les composantes du milieu affectées par les activités agricoles. L'impact des sous activités de l'agriculture a été décrit par rapport à la nature, la durée, la portée (étendue) et l'intensité sur chaque composante du milieu affecté. L'analyse et l'évaluation de l'importance des impacts reposent sur une approche méthodologique qui intègre les trois paramètres de l'impact à savoir la durée (courte, moyenne ou longue), l'étendue (ponctuelle, locale ou régionale) et l'intensité (faible, moyenne ou forte). Une autre approche méthodologique qui intègre les trois paramètres de l'impact à savoir la durée (momentanée, temporaire et permanente), l'étendue (ponctuelle, locale ou régionale) et le degré de perturbation (faible, moyen, fort et très fort) est celle de l'ABE (1999). Concernant l'impact environnemental des activités agricoles sur les écosystèmes et les conditions de vie des populations rurales, les valeurs environnementales des différentes composantes affectées ont été évaluées.

Analyse des changements sociaux: L'analyse des changements sociaux, a permis de distinguer les changements qui représentaient des processus et des événements sociaux qui étaient des phonèmes 
ponctuels. II n'a pas été question d'analyser tout ce qui était apparu comme nouveau dans l'agriculture du nord-ouest de l'Atacora. II a été plus mis l'accent sur les processus pertinents ayant eu un impact sur la communauté rurale de la zone d'étude. II s'était intéressé de savoir ce qui a changé des années 1993 à 2013 et à voir si ce changement était repérable en raison de la situation de référence caractérisée par un très faible développement du rendement des céréales par exemple et une production cotonnière plus raisonnée. Des cas où, les causes lointaines de ces changements persistaient, elles ont été rappelées et analysées. Le repère des années 90 s'expliquait par le fait qu'elles ont été déterminantes pour le pays (ajustement structurel) comme sur le plan international (sommet de Rio, globalisation du discours communautaire et participatif, gouvernance locale). La durabilité des transformations structurelles induites a été prise en compte, ce qui poussait à ne parler de changement social qu'après avoir prouvé la durabilité des modifications étudiées. Etant entendu que le changement social était un phénomène collectif, il a été cherché à vérifier s'il touche toute la communauté étudiée au groupe socioculturel, une organisation, un village, une institution locale donnée ou des individus pris dans leur ensemble. A cet égard, les facteurs de changement qui ont été considérés étaient les valeurs culturelles ou les progrès techniques (promotion du coton) et la démographie (accroissement de la population). Pour le dernier cas de facteurs, il a été utilisé les informations statistiques des recensements

\section{RESULTATS}

Evolution des superficies emblavées et de la production des principales spéculations entre 1993 et 2013 : L'évolution des superficies emblavées et de la production des principales spéculations du secteur d'étude est présentée sur les figures 2 à 9 . Ces principales spéculations sont regroupées en les quatre catégories suivantes: les céréales; les légumineuses ; les racines et tubercules ; le coton. généraux de la population et de l'habitation de 1992, 2002 et 2013 complétées par des enquêtes sur le terrain.

Diagnostic : Sur le terrain, le diagnostic s'était déroulé en deux phases: une première phase qui a consisté à explorer et réaliser la typologie des exploitations agricoles en ce qui concerne la gestion de la fertilité des sols et une seconde phase d'enquêtes complémentaires sur les caractéristiques des exploitations types selon les classes de gestion de la fertilité des sols. Ces phases ont été exécutées entre 2011 et 2013. La typologie des exploitations suivante a été réalisée lors des réunions villageoises au cours desquelles des « focus group » ont été effectués:

- la hiérarchisation des pratiques et facteurs déterminants de la gestion de la fertilité des sols au niveau des exploitations dans les villages;

- la typologie des exploitations agricoles en trois (03) classes selon des critères basés sur les pratiques et facteurs déterminants.

Cette phase du diagnostic a été réalisée sur un échantillon de 400 exploitations agricoles (soit $5 \%$ des villages et des ménages ruraux) réparties dans huit (08) villages de la zone d'étude. Les résultats d'une autre étude diagnostique réalisée sur la gestion de la fertilité des sols conduite dans $10 \%$ au moins des villages de chaque Commune en 2012. Les enquêtes complémentaires ont été effectuées auprès d'exploitants représentatifs des trois (03) classes.

Evolution des superficies emblavées et de la production des céréales entre 1993 et 2013: L'évolution des superficies emblavées et de la production pour les principales cultures céréalières au nord-ouest de l'Atacora entre 1993 et 2013 est présentée sur les figures 2; 3 . 


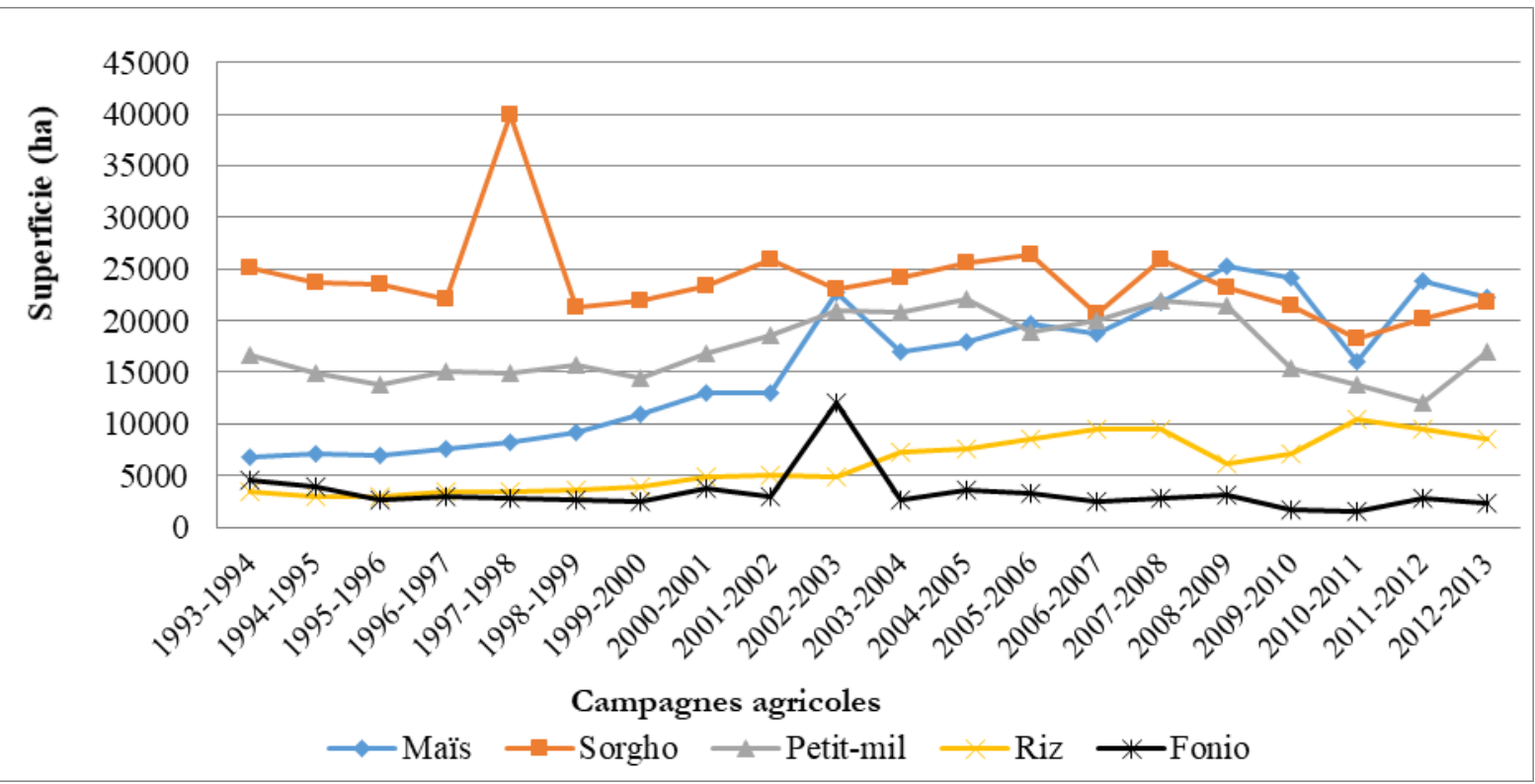

Figure 2: Superficies emblavées pour les céréales entre 1993 et 2013 Source : CeRPA-Atacora-Donga, 2013

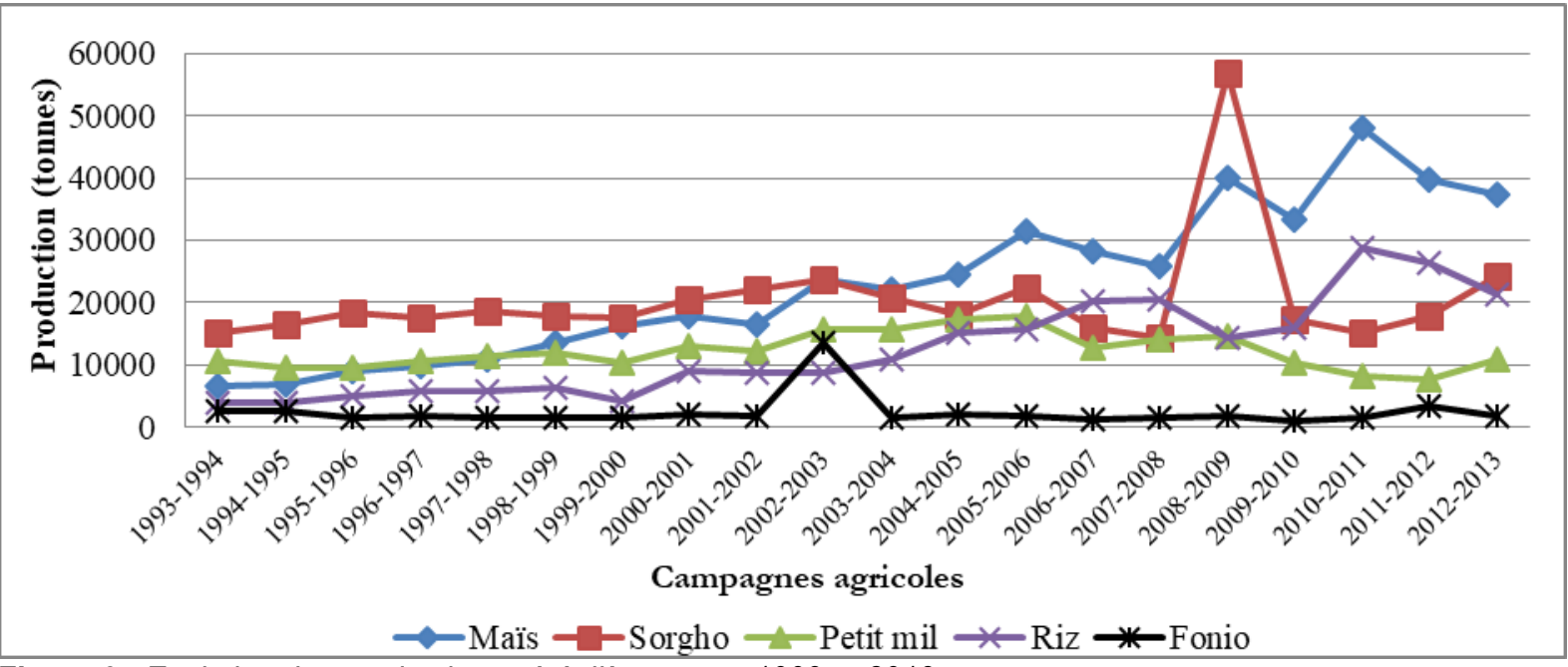

Figure 3 : Evolution des productions céréalières entre 1993 et 2013

Source : CeRPA-Atacora-Donga, 2013

Le maïs, le sorgho, le petit mil, le riz et le fonio ont été les cultures céréalières les plus importantes inventoriées entre 1993 et 2013 au nord-ouest de l'Atacora (Figure 2). Les superficies emblavées pour les différentes spéculations variaient dans le temps. Ainsi, le sorgho qui était la principale spéculation des populations du nord-ouest de l'Atacora de 1993 (25.153 ha) à 2008 (25.895 ha) était progressivement devenu la deuxième spéculation en termes d'emblavures de 2009 (23.146 ha) à 2013 (21.788,8 ha). Au cours de la période 1993-2013, la variation des superficies emblavées pour le sorgho avait connu une évolution en dents de scie avec un important pic (39.853 ha) au cours de la campagne 1997-1998. Par ailleurs, le maïs qui occupait la troisième place en termes d'utilisation des terres de 1993 (6.736 ha) à 2007 (18.666 ha) était devenu la première spéculation de 2009 (25.254 ha) à 2013 (21.788,8 ha). Au cours de la période 1993-2013, l'augmentation des superficies emblavées pour le maïs n'a pas été régulière. Elle avait connu un pic (23.022 ha) et une chute (16.073 ha) respectivement au cours des campagnes 2002-2003 et 2010-2011. Le petit mil était passé de la deuxième place entre 1993 (16.689 ha) et 2008 (21.967 ha) à la troisième place à partir de 2009 (21.516 ha). Les superficies emblavées pour le petit mil ont connu une régulière augmentation jusqu'en 
2009 avant de chuter (Figure 2). Les superficies emblavées pour le riz ont connu une régulière augmentation de 1993 (4.544 ha) à 2013 (8.513,4 ha). De 1996 à 2013, hormis la campagne 2002-2003, le riz était devenu la quatrième spéculation la plus importante en termes d'utilisation des terres. Par contre, les superficies emblavées pour le fonio étaient restées stables de 1993 à 2013 à l'exception de la campagne 2002-2003 où une emblavure record de 12.021 ha a été obtenue. Les emblavures du fonio ont été les plus faibles parmi les spéculations céréalières les plus importantes dans le nord-ouest de l'Atacora entre 1993 et 2013 (Figure 2). Les productions des différentes céréales ont globalement connu une augmentation entre 1993 et 2013 (Figure 3). Cependant, quelques spécificités étaient à signaler d'une spéculation à une autre. Le sorgho était la principale spéculation des populations du nord-ouest de l'Atacora de 1993 (15.294 t) à $2003(23.800 \mathrm{t})$. Avec la baisse de sa production, elle était progressivement devenue la deuxième spéculation de 2004 (20.869 t) à 2006 (22.478 t) et au cours des campagnes 2009-2010 (15.996 t) et 20122013 (24.278,8 t). De 2007 à 2012, le sorgho était devenu la troisième spéculation en termes de production à l'exception de la campagne 2008-2009 où cette production avait connu une augmentation exponentielle avec plus de 56.784 tonnes reprenant du coup la première place. La production du maïs était passée de la troisième place entre 1993 (6.488 t) et
1998 (11.437 t) à la deuxième place entre 1999 (13.526 t) et 2003 (23.800 t). De 2003 à 2013, hormis la campagne 2008-2009, la production du maïs était devenue la première spéculation avec une production maximale de $48.061 \mathrm{t}$ au cours de la campagne 20102011. Cette évolution de la production s'était faite en dents de scie durant la période considérée (figure 3).La production du petit mil avait connu une régulière augmentation de 1993 à 2006. Au cours de cette période, elle était passée de la deuxième place entre $1993(10.572 \mathrm{t})$ et 1998 (11.437 t) à la troisième place de 1999 (12.045 t) à 2006 (17.914 t). De 2007 (12.874 t) à 2013 (11.019 t), la production de cette céréale avait baissé, l'entraînant de ce fait à la quatrième place (figure 3). La production du riz avait connu une augmentation régulière de 1993 (3.885 t) à 2008 $(20.455 \mathrm{t})$ avant de chuter (14.311 t) au cours de la campagne agricole 2008-2009. Une reprise a été notée à partir de la campagne 2009-2010 avec un pic (28.840 t) en 2010-2011.Enfin, la production du fonio était demeurée stable durant toute la période à l'exception de la campagne 2002-2003 où la production avait atteint $13.498 \mathrm{t}$.

Evolution des superficies emblavées et de la production des légumineuses entre 1993 et 2013 : L'évolution des superficies emblavées et de la production pour les légumineuses entre 1993 et 2013 est présentée sur les figures $4 ; 5$.

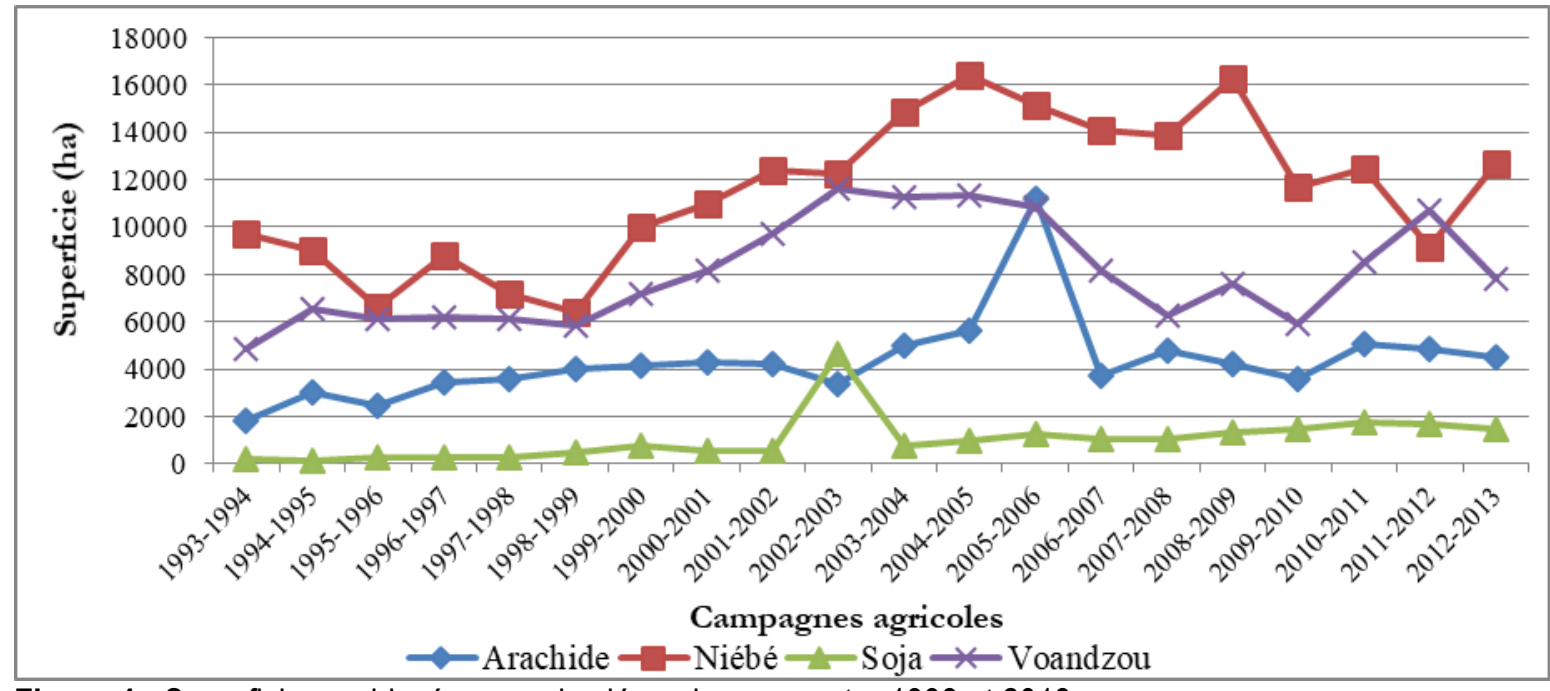

Figure 4 : Superficies emblavées pour les légumineuses entre 1993 et 2013 Source : CeRPA-Atacora-Donga, 2013 


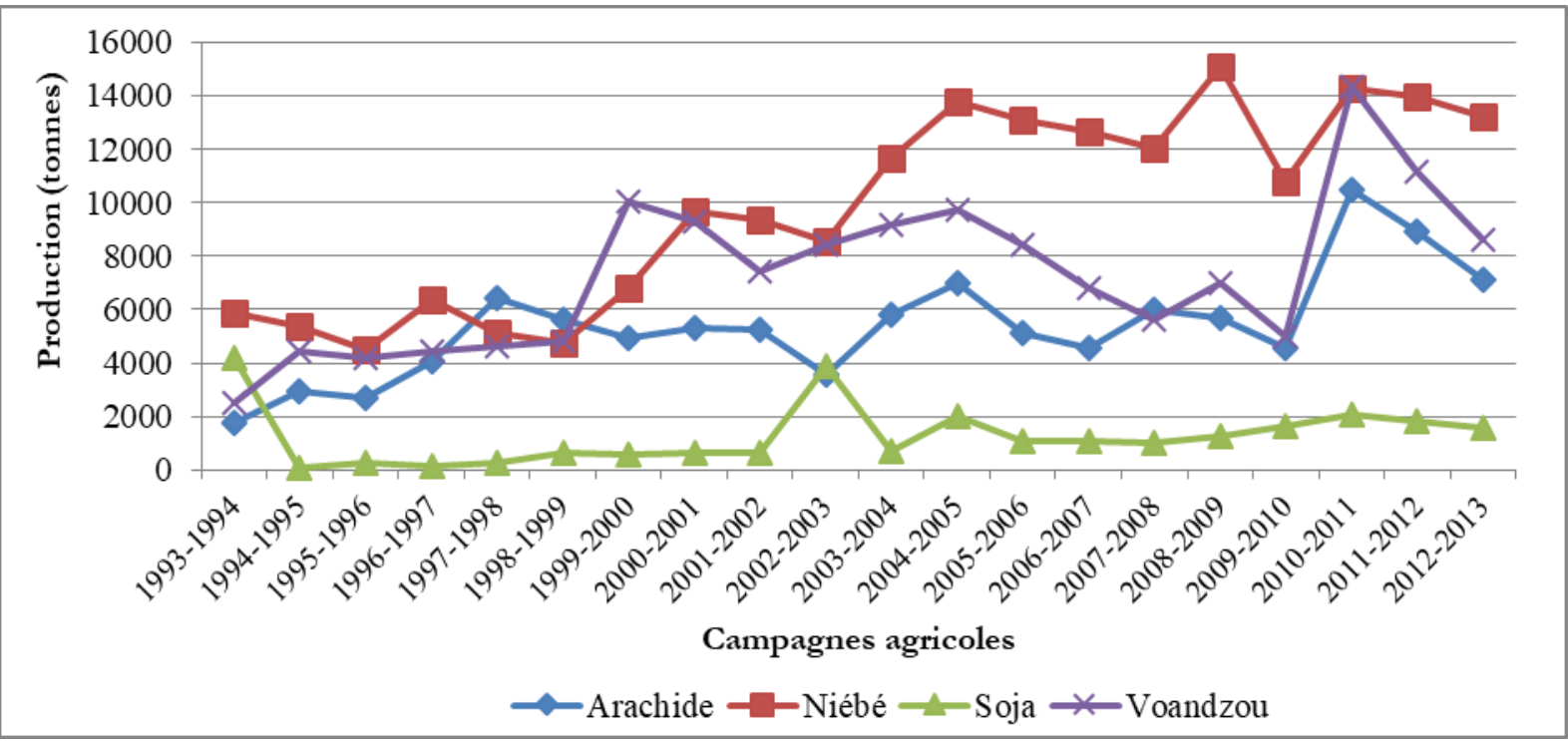

Figure 5 : Evolution de la production des légumineuses entre 1993 et 2013

Source : CeRPA-Atacora-Donga, 2013

Le niébé, le voandzou, l'arachide et le soja sont les légumineuses les plus importantes inventoriées au nord-ouest de l'Atacora entre 1993 et 2013. Les superficies emblavées pour le niébé et le voandzou ont varié en dents de scie (Figure 4). Par contre, celles de l'arachide et du soja ont connu une certaine régularité en dehors d'un pic qui a été obtenu respectivement au cours de la campagne 2002-2003 pour le soja et de la campagne 2005-2006 pour l'arachide. Les superficies emblavées pour le niébé ont évolué en dents de scie avec le maximum d'emblavure (16.254 ha) au cours des campagnes 2004-2005 et 2008-2009. Le voandzou a été la deuxième spéculation de légumineuses du secteur d'étude. Les superficies emblavées pour cette spéculation ont évolué en dents de scie avec le maximum d'emblavure au cours de la campagne 20022003 (11.615 ha). Cette courbe des superficies emblavées pour le voandzou a présenté de 1993 à 2006, deux phases ascendantes (1993-1995; 19992003) et deux phases de stabilisation (1995-1999; 2003-2006). De 2006 à 2013, les superficies emblavées ont drastiquement chuté avec une légère reprise (10.720 ha) au cours de la campagne 20102011 puis une rechute (7.792 ha) au cours de la campagne 2012-2013. L'arachide a été la troisième spéculation en termes de superficies emblavées dans le secteur d'étude. Toutefois, elle a été supplantée par l'emblavure du soja au cours de la campagne 20052006 (3.371 ha). Par ailleurs, c'était au cours de la campagne 2005-2006 que le maximum d'emblavure (11.177 ha) a été réalisé pour l'arachide en léguant de ce fait le voandzou à la troisième place. Enfin, les superficies emblavées pour le soja ont régulièrement augmenté en passant de 224 ha en 1993 à 1.462,6 ha en 2013. Durant cette période, les superficies ont connu une augmentation brutale (4.636 ha) au cours de la campagne 2002-2003. La production des légumineuses a globalement connu une augmentation entre 1993 et 2013 (Figure 5). Cependant, quelques spécificités étaient à signaler d'une culture à une autre. La production du niébé a connu une évolution en dents de scie de 1993 à 2013. Le niébé était la principale spéculation des populations du nord-ouest de l'Atacora de 1993 (5.862 t) à $2013(13.238,6 t)$ en dehors des campagnes 1997-1998 et 1998-1999 où il a été supplanté par l'arachide et de la campagne 1999-2000 qui était dominée par le voandzou. La production maximale (15.060 t) de cette spéculation a été obtenue au cours de la campagne 2008-2009. Le taux d'accroissement de cette spéculation était de 55,72\% durant la période d'étude. L'augmentation de la production du voandzou n'a pas été régulière. Elle a connu des pics et des chutes durant la période d'étude. Elle était passée de 2.510 t en 1993 à $8.635,6$ t en 2013 soit un taux d'accroissement de 70,93\%. La production record de 14.343 tests réalisés au cours de la campagne 2010-2011. Cette spéculation était passée de la troisième place au cours des campagnes 19931994 et 1997-1998 à la première place entre 1999 et 2000 avant de rechuter à la deuxième place dans la production des légumineuses. La production d'arachide était passée de 1.782 t en 1993 à 7.125,6 t en 2013 soit un accroissement de près de $75 \%$. La production record de 10.480 t a été » obtenue au cours de la campagne 2010-2011. Cette spéculation était passée de la quatrième place en 1993 à la première place en 
1998 avant de rechuter à la troisième place. Enfin, la production du soja a chuté de la deuxième place en 1993 (4.201 t) à la quatrième place de 1995 à 2013 $(1.567,2$ t). Soit une régression de $62,69 \%$. Cette production était demeurée stable durant toute la période (1995-2013) à l'exception de la campagne 2002-2003 où la production a atteint $3.873 \mathrm{t}$ (Figure 5).
Evolution des superficies emblavées et de la production des racines et tubercules entre 1993 et 2013 : L'évolution des superficies emblavées et de la production pour les racines et tubercules entre 1993 et 2013 est présentée sur les figures $6 ; 7$.

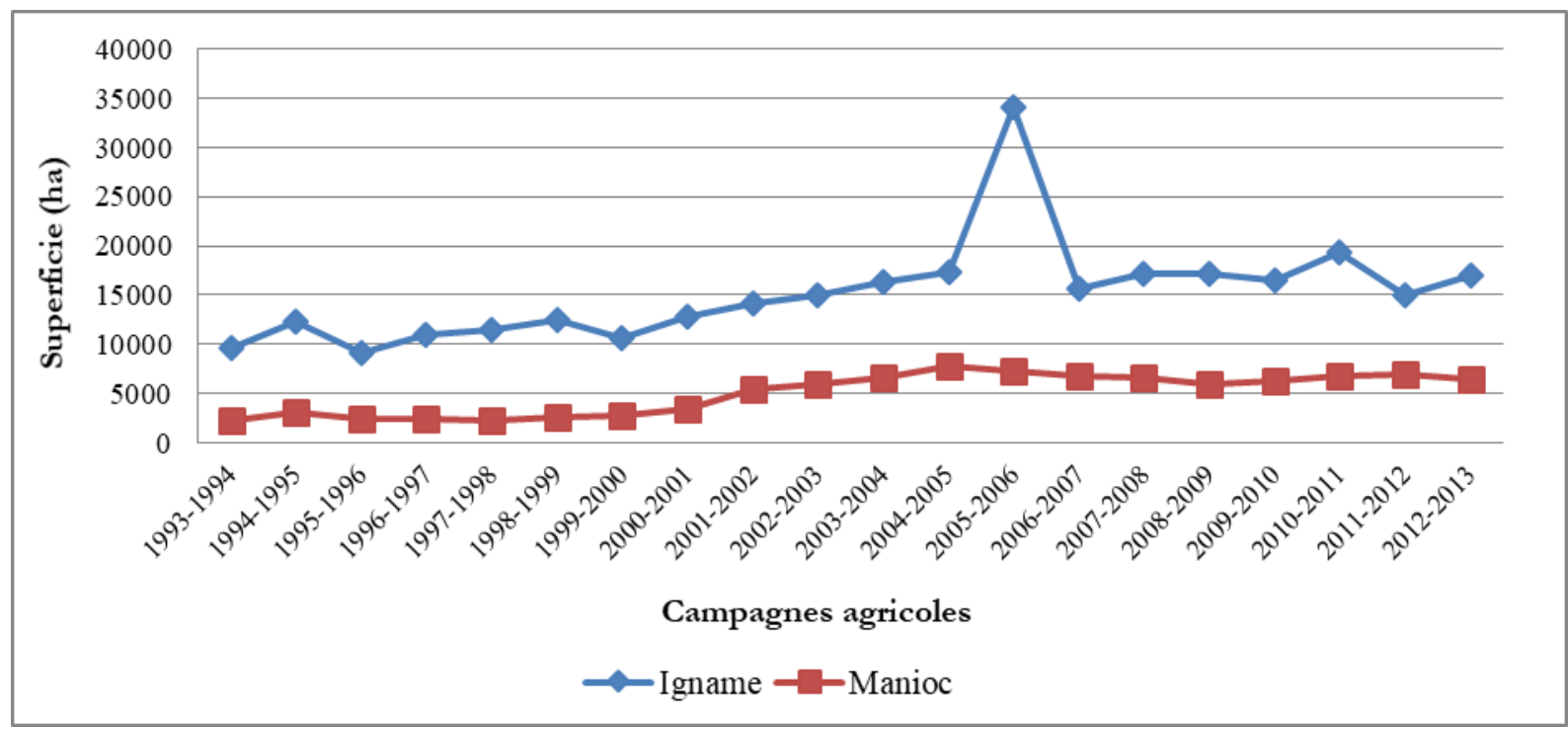

Figure 6 : Superficies emblavées pour les racines et tubercules entre 1993 et 2013

Source : CeRPA-Atacora-Donga, 2013

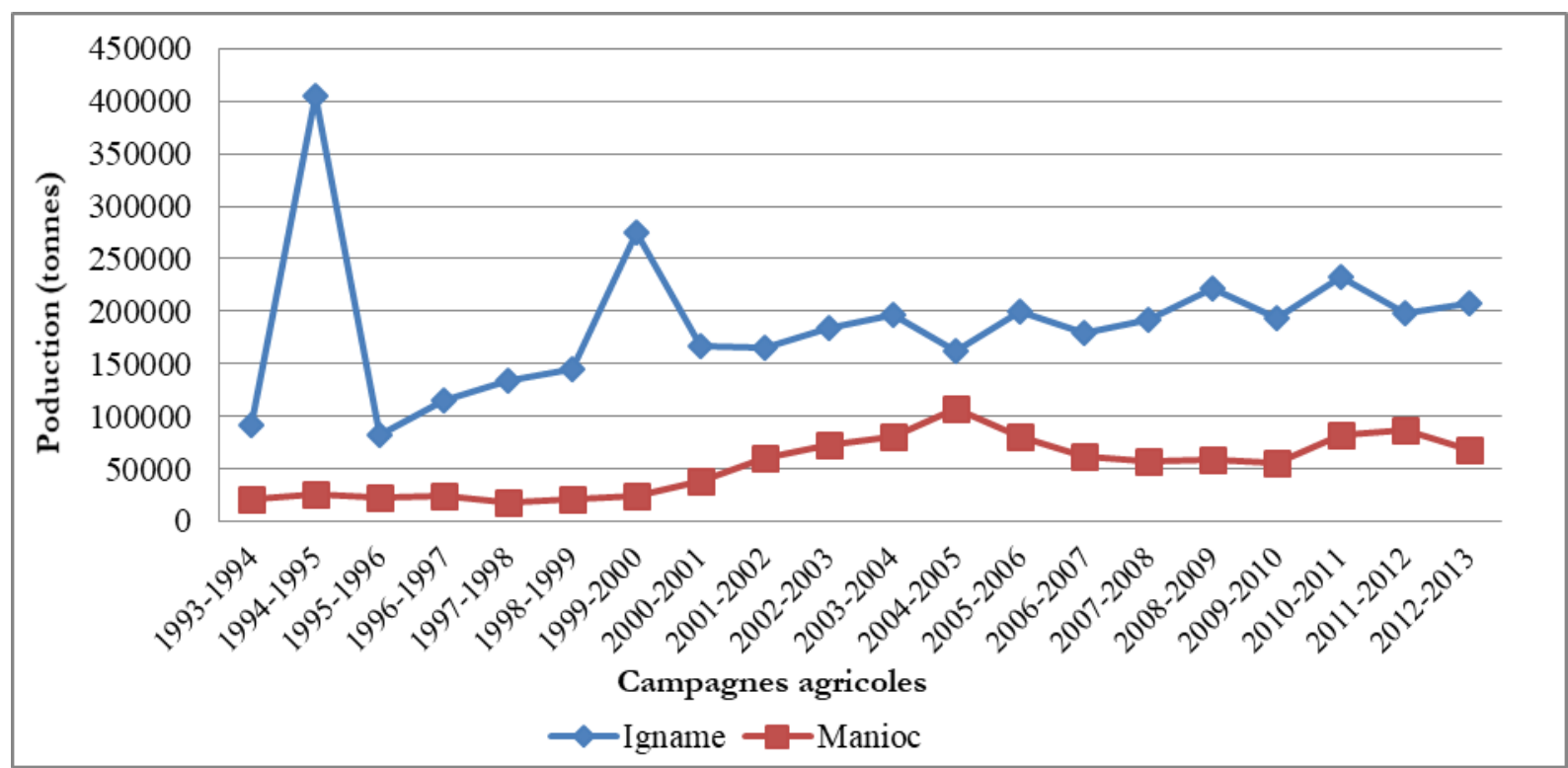

Figure 7 : Evolution de la production des racines et tubercules entre 1993 et 2013 Source : CeRPA-Atacora-Donga, 2013

Le manioc et l'igname ont été respectivement les principales racines et tubercules des populations du nord-ouest de l'Atacora. Les superficies emblavées pour l'igname étaient plus importantes que pour le manioc. Les superficies emblavées pour l'igname étaient passées de 9.626 ha au cours de la campagne 1993-1994 à 17.071,8 ha pour la campagne 2012-2013 avec un pic de 34.067 ha au cours de la campagne 
2005-2006 (Figure 6). Par ailleurs, les superficies emblavées pour le manioc étaient passées de 2.304 ha au cours de la campagne 1993-1994 à 6.470,8 ha pour la campagne 2012-2013. Durant la période d'étude, les plus grandes superficies emblavées pour le manioc (7847 ha) ont été réalisées au cours de la campagne 2004-2005. La production réalisée par les principales cultures des racines et tubercules entre 1993 et 2013 était passée de $91.126 \mathrm{t}$ à $207.426 \mathrm{t}$, soit une augmentation de $56,06 \%$ en 20 ans. La production de l'igname et du manioc entre 1993 et 2013 est présentée sur la figure 7 . La production de l'igname était plus importante que celle du manioc au nord-ouest de l'Atacora entre 1993 et 2013. L'igname a connu une production record de 405.774 t en 1995. Le deuxième pic de la production au cours de la période 1993-2013 a été de 275.648 t en 2000.La production du manioc n'a jamais atteint les $100.000 \mathrm{t}$ à l'exception de la campagne 2004-2005 où elle était de 107.287 t (Figure 7).

Evolution des superficies et de la production du coton entre 1993 et 2013 : L'évolution des superficies emblavées et de la production cotonnière entre 1993 et 2013 est présentée sur les figures $8 ; 9$.

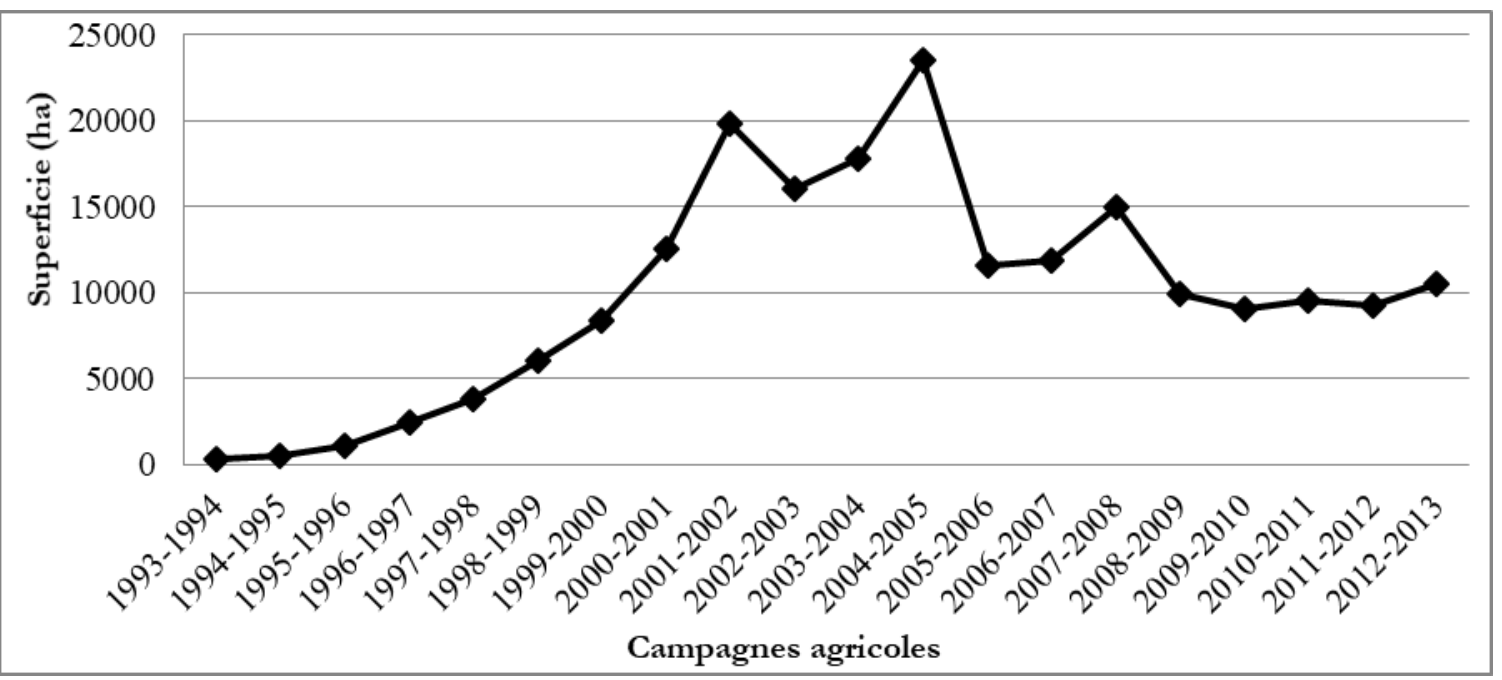

Figure 8 : Superficies emblavées pour le coton entre 1993 et 2013

Source : CeRPA-Atacora-Donga, 2013

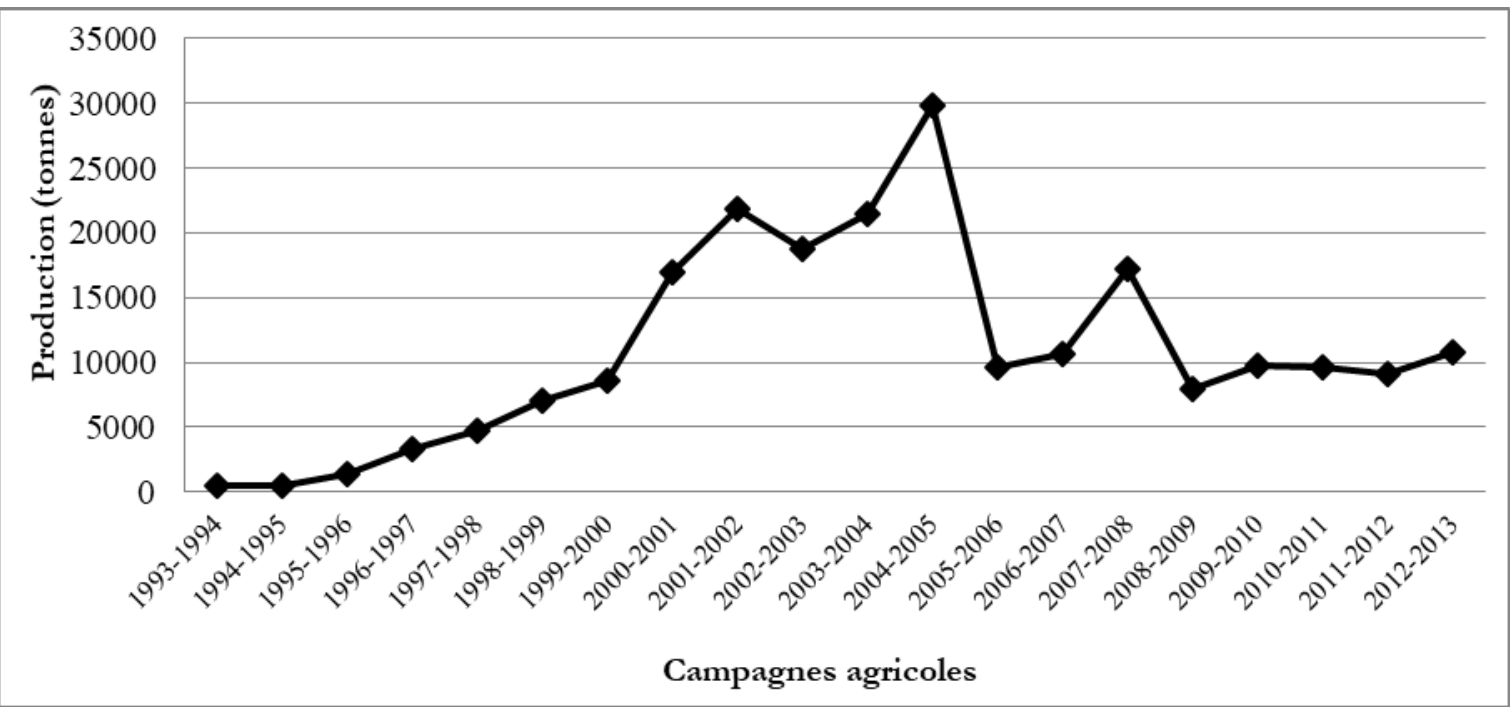

Figure 9 : Evolution de la production du coton entre 1993 et 2013

\section{Source : CeRPA-Atacora-Donga, 2013}

Les superficies emblavées pour le coton évoluent de façon régulière entre 1993 (340 ha) et 2002 (19.848 ha) avec un premier pic au cours de la campagne 20012002. De 2002 à 2010, les superficies emblavées 
évoluent en dents de scie avec deux pics au cours des campagnes 2004-2005 (23.500 ha) et 2007-2008 (14.961 ha). De 2010 à 2012, une phase de stabilité est observée avant une timide reprise en 2013 (10.562,2 ha) (Figure 8). La production du coton a évolué de $473 \mathrm{t}$ en 1993 à 10.738 en 2012 (Figure 9). Ce qui correspondait à une augmentation de la production de 95,59\%. Elle a évolué de façon progressive entre 1993 et 2001. Après des pics au cours des campagnes agricoles 2001-2002 et 2004-2005, elle a connu une chute importante au cours des campagnes agricoles 2002-2003 et 2005-2006 même si un léger pic a été constaté au cours de la campagne agricole de 20072008.

Incidences socio-environnementales des activités agricoles : Le nord-ouest de l'Atacora était une zone dont le milieu naturel a été soumis à une forte pression de ses habitants qui tiraient l'essentiel de leurs revenus des activités agricoles. Les impacts de la production agricole se traduisaient surtout entre autres, par la disparition du couvert végétal, la baisse de la fertilité des sols et la pollution de certains cours d'eau aux bords desquels se réalisaient des activités à dominance agricole. L'évaluation des impacts s'était reposée sur l'approche méthodologique de l'Agence Béninoise pour l'Environnement (ABE) dont la synthèse a été mentionnée dans les tableaux 1 et 2. De façon pratique, il a été identifié d'abord l'état (situation de base existante avant l'intervention des facteurs de pressions) ensuite les pressions (forces agissantes et ayant induit des changements dans un état habituel), les impacts (dommages et risques dus aux changements intervenus) et leurs réponses (mesures correctrices pour atténuer les impacts négatifs et maximiser ceux positifs) (Figure 11). Ce modèle permet de caractériser à partir d'un état initial les impacts des forces motrices qui ont exercé une certaine pression sur les composantes de l'environnement au nord-ouest de l'Atacora (Figure 10). 
Tableau 1 : Matrice d'évaluation des impacts liés à l'exploitation agricole sur le milieu physique

\begin{tabular}{|c|c|c|c|c|c|c|}
\hline $\begin{array}{l}\text { Sources d'impacts } \\
\text { Activités }\end{array}$ & $\begin{array}{l}\text { Composantes } \mathrm{du} \\
\text { milieu affectées }\end{array}$ & Impacts & Nature & Durée & Etendue & $\begin{array}{l}\text { Degré } \\
\text { perturba }\end{array}$ \\
\hline Défrichement & Sol & Dégradation & Négative & Permanente & Locale & Moy \\
\hline \multirow[b]{2}{*}{ Ecobuage } & & $\begin{array}{l}\text { Dégagé pour les } \\
\text { semis }\end{array}$ & Positive & Permanente & Locale & \\
\hline & Sol & $\begin{array}{l}\text { Destruction des } \\
\text { éléments nutritifs }\end{array}$ & Négative & Permanente & Locale & \\
\hline \multirow[b]{2}{*}{ Utilisation des intrants chimiques } & Végétaux & $\begin{array}{l}\text { Accumulation de } \\
\text { métaux } \\
\text { lourds }\end{array}$ & Négative & Permanente & Locale & \\
\hline & Sol & $\begin{array}{l}\text { Accumulation de } \\
\text { métaux lourds }\end{array}$ & Négative & Permanente & Locale & \\
\hline
\end{tabular}

Tableau 2: Matrice d'évaluation des impacts liés à l'exploitation agricole sur le milieu biologique et les producteurs

\begin{tabular}{|c|c|c|c|c|c|c|}
\hline $\begin{array}{l}\text { Sources d'impacts } \\
\text { Activités }\end{array}$ & $\begin{array}{l}\text { Composantes du } \\
\text { milieu affectées }\end{array}$ & Impacts & Nature & Durées & Etendue & $\begin{array}{l}\text { Degré } \\
\text { perturba }\end{array}$ \\
\hline \multirow{3}{*}{$\begin{array}{l}\text { Défrichement/ } \\
\text { Feux de végétation et chasse à la } \\
\text { battue }\end{array}$} & $\begin{array}{l}\text { Couvert végétal et } \\
\text { rochers }\end{array}$ & $\begin{array}{l}\text { Destruction du } \\
\text { couvert végétal }\end{array}$ & Négative & Temporaire & Locale & Moye \\
\hline & Faune & $\begin{array}{c}\text { Risque de disparition } \\
\text { des animaux ayant } \\
\text { pour abris les } \\
\text { végétaux détruits }\end{array}$ & Négative & Temporaire & Locale & Moye \\
\hline & $\begin{array}{c}\text { Acteurs de } \\
\text { l'exploitation agricole } \\
\text { et habitants }\end{array}$ & $\begin{array}{l}\text { Augmentation des } \\
\text { revenus }\end{array}$ & Positive & Temporaire & Locale & Moye \\
\hline \multirow{2}{*}{$\begin{array}{l}\text { Utilisation } \\
\text { des engrais chimiques }\end{array}$} & & $\begin{array}{l}\text { Amélioration des } \\
\text { rendements }\end{array}$ & Positive & Temporaire & Locale & Moye \\
\hline & $\begin{array}{c}\text { Acteurs de } \\
\text { l'exploitation agricole }\end{array}$ & $\begin{array}{c}\text { Augmentation } \\
\text { précaire des revenus }\end{array}$ & $\begin{array}{l}\text { Positive/ } \\
\text { Négative }\end{array}$ & Permanente & Locale & Moye \\
\hline Fabrication de charbon & $\begin{array}{l}\text { Fabricants et } \\
\text { populations } \\
\text { environnantes }\end{array}$ & $\begin{array}{l}\text { Risque de pollution } \\
\text { de l'environnement }\end{array}$ & Négative & Temporaire & Régionale & $\overline{M o}$ \\
\hline
\end{tabular}


La nature des impacts négatifs a prédominé ceux positifs (tableaux 1 et 2). Certaines activités telles que l'utilisation des engrais chimiques et les feux de végétation ont résolu d'une manière ponctuelle certains problèmes comme l'amélioration des rendements agricoles et l'augmentation des revenus, mais elles n'ont pas duré dans le temps. Au fil des années, ces activités ont impacté négativement le milieu et les conditions de vie des populations. II est important de trouver des alternatives sûres, car les impacts négatifs semblent devenir permanents dans le secteur d'étude.

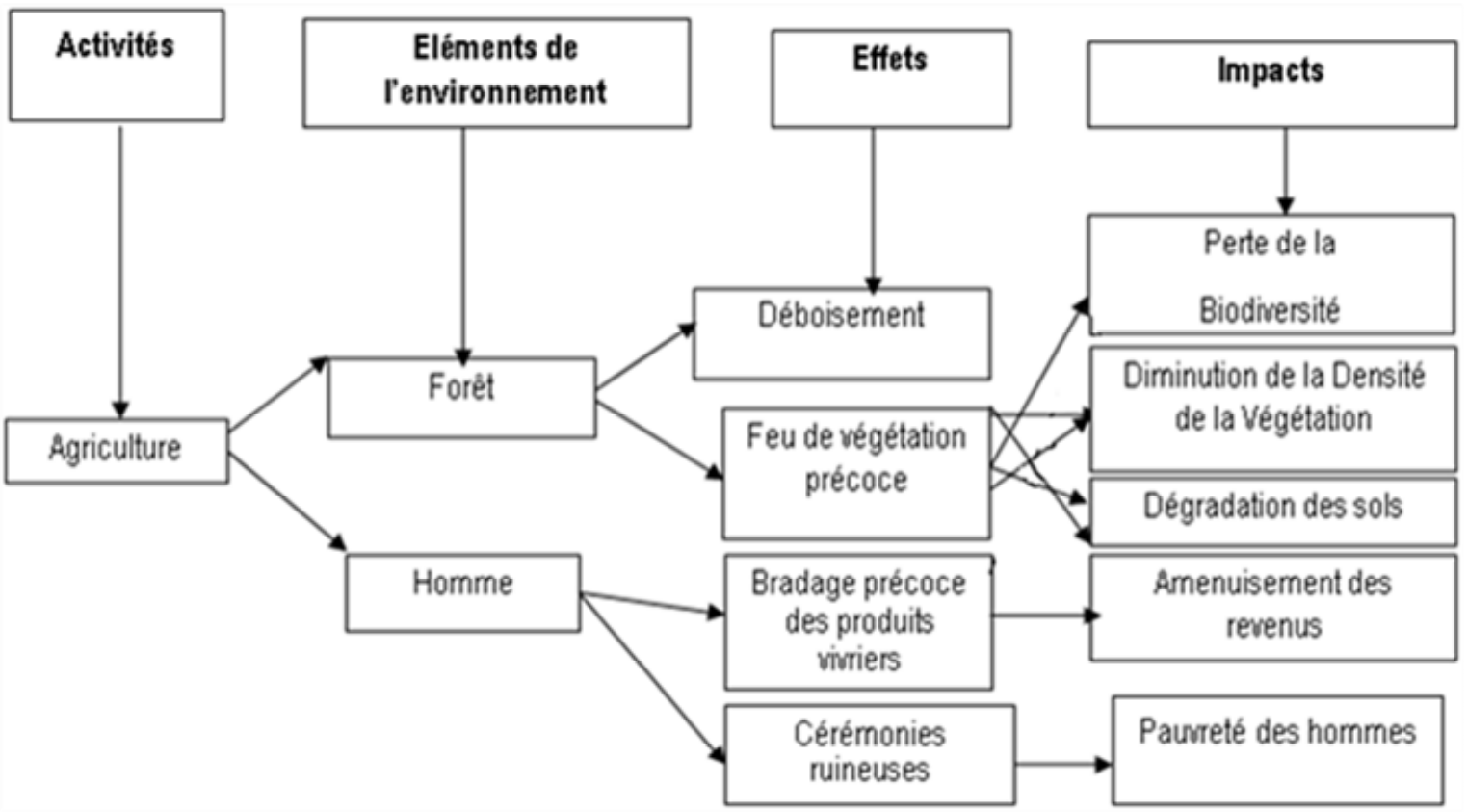

Figure 10: Interactions entre activités, éléments, effets et impacts environnementaux

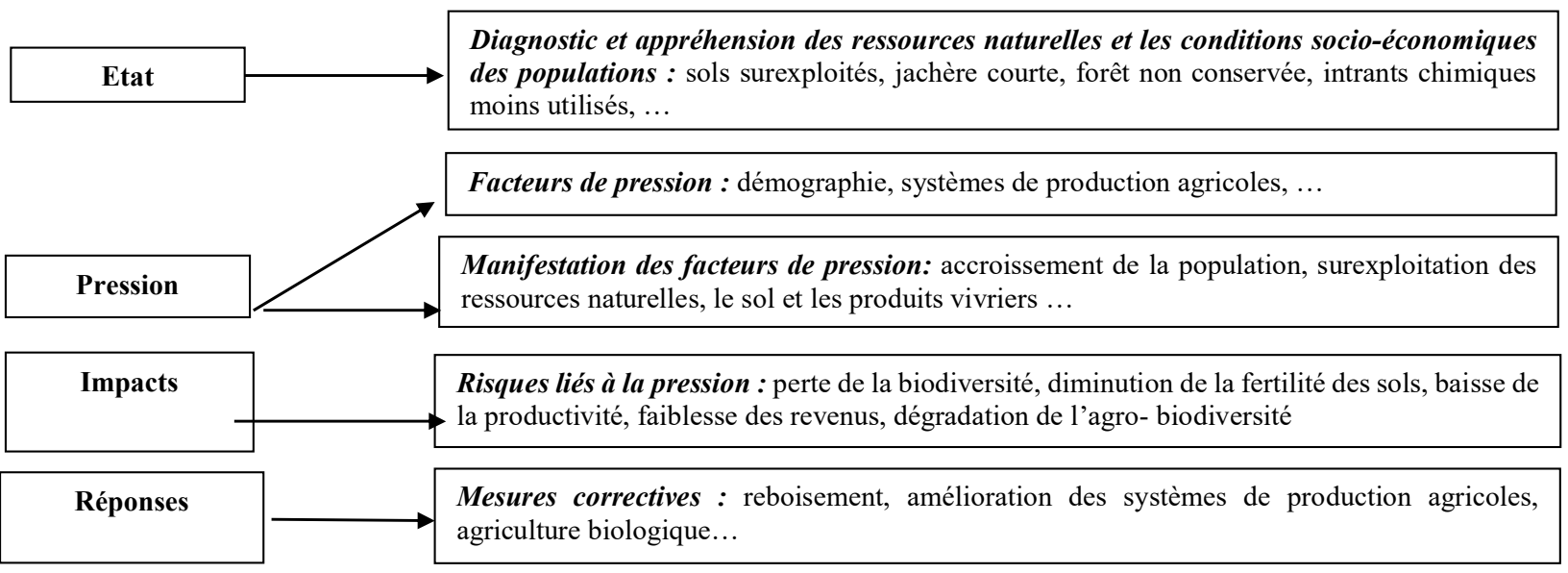

Figure 11 : Analyse des résultats à l'aide du PEIR

\section{DISCUSSION}

Impacts socio-environnementaux des activités agricoles au nord-ouest de l'Atacora : Les pratiques des producteurs enquêtés en ce qui concerne l'exécution des activités agricoles et des techniques mises en œuvre montrent que tout concoure à la satisfaction des besoins alimentaires. Cependant, de nombreuses pratiques viennent aggraver l'impact sur l'environnement. En effet, la dégradation des ressources naturelles (sols, végétation) est en grande partie la conséquence du système agricole. L'agriculture itinérante sur brûlis liée surtout à la culture de l'igname et celle du coton constitue la cause essentielle des défrichements sur de vastes étendues (Kombienou, 2016). En plus, le non-respect des doses d'engrais chimiques ou la non utilisation de fertilisants conduit à un appauvrissement prolongé des terres à 
cause des manques d'éléments nutritifs (Boli et al., 1993; Azontondé, 2000 ; Mulder, 2000; Akissoé et al., 2001 et Kombienou et al., 2015). De plus, l'exploitation prolongée des mêmes terres entraîne une dégradation poussée des sols. Les pratiques culturales inappropriées dégradent les sols et les systèmes de culture sans amendement réduisent considérablement les teneurs en matière organique et de l'azote (Azontondé et al., 1998 et Koundé, 1998). Ce qui empêche la régénération des arbres et arbustes, appauvrit le stock de matières organiques du sol et favorise l'érosion. De même, l'exploitation des flancs des montagnes en déterrant certaines pierres fait exposer le sol à l'érosion hydrique et éolienne. Les sols sont graveleux et rocailleux. L'eau y ruisselle et érode les sols encore cultivables qui se réduisent peu à peu. II faut noter que les feux de végétation appauvrissent les sols en humus comme l'a prouvé une étude réalisée au Kenya pendant dix ans sur plusieurs parcelles (Ramade, 1991). La première parcelle protégée a enregistré un taux d'accroissement de matière humique, contrairement aux autres parcelles, annuellement parcourues par les feux. La conséquence, le sol est mis à nu et exposé à l'action destructrice des gouttes de pluie et des eaux de ruissellement. Les sols sont privés d'une grande partie de la végétation pouvant leur fournir de la matière organique suite à leur décomposition. Selon Arouna (2005 et 2012) et Kombienou et al. (2016), la pratique des défrichements culturaux dénude le sol et le prive durant une période de l'année de son couvert végétal protecteur. Le sol mis à nu est décapé par les gouttelettes de pluies qui dissocient les particules de sols en les entrainant par l'eau qui les dévale (Djaouga, 2003). Les fortes pressions sur les terres par la réduction de la période de jachères dégradent davantage les sols qui sont soumis à l'érosion et à l'appauvrissement. Dans le même ordre d'idées, Démon (1991) affirme que la perte de la couverture végétale et l'appauvrissement des sols relèvent des activités humaines. Les techniques et les modes d'exploitations utilisées pour mener les activités au nord-ouest de l'Atacora ne tiennent donc pas compte

\section{CONCLUSION}

L'association des cultures est le système de cultures le plus utilisé dans les agro-systèmes du nord-ouest de l'Atacora. L'association, sorgho-mil est une pratique courante chez les Bètammaribè et les Waaba qui pratiquent, en plus, l'association céréales-niébé (haricot). L'association sorgho-niébé à port étalé par exemple permet de lutter contre les mauvaises herbes et améliore la qualité du sol. Pourtant, l'association de de la préservation de l'environnement. Du fait des pratiques agricoles et de la pression démographique, le sol, principal support de cette agriculture connaît des dégradations qui affectent sa fertilité (Natta, 1999). Dans ce contexte de dégradation du potentiel productif des sols vient s'ajouter celle de la variabilité climatique qui a des conséquences négatives sur la production agricole (Ouorou Baré, 2014). Avec une population mondiale qui devrait atteindre 9 milliards d'ici 2050, le sol est devenu une ressource fondamentale à protéger de toute urgence (FAO, 2003 ; ISRIC, 2007).En somme, la dégradation des sols est préoccupante, les érosions éoliennes et pluviales éliminent la mince couche d'humus. Elles déchaussent les arbustes et les arbres qui n'ont pas de profondes racines. La plupart de ces sols ont besoin aujourd'hui d'être protégés contre l'érosion par des plantations d'arbres et la mise en place de petits ouvrages antiérosifs. Ces sols ont aussi besoin d'importants apports en matières organiques pour la reconstruction de la couche humifère sur les parcelles mises en culture (Zinzindohoué, 2012). Les problèmes liés à l'exploitation de ces sols, leur surexploitation (due aux associations complexes des cultures, au manque et à l'insuffisance des jachères, à la pression démographique), l'érosion et la baisse de fertilité nécessitent l'adoption de pratiques culturales performantes et adaptées pour la promotion de l'agriculture. Ce qui amène les chercheurs à proposer des solutions dans le cadre de l'amélioration du taux de la matière organique par une jachère de Mucuna qui améliore de 4 à 5 fois la productivité que l'engrais chimique (Honlonkou, 1999). De même, l'enlèvement de la matière organique que constitue le départ de la couche fertile entraine une diminution de la capacité de rétention des eaux de pluie et une augmentation du ruissellement. Dans les conditions pluviométriques actuelles, il faut, pour optimiser la production agricole, favoriser l'infiltration de l'eau dans le sol et sa rétention maximale grâce aux apports de matières organiques tels que : le fumier, le compost et l'engrais vert (Adewi, 2012).

certaines cultures présente des inconvénients, c'est le cas par exemple de l'association céréales-céréales et tubercules-céréales qui augmentent la compétitivité entre les plantes de même famille, surtout sur des sols déjà très pauvres. Les céréales (sorgho, petit mil, fonio, riz et maïs), les tubercules (ignames et taro) et les légumineuses (niébé, voandzou et arachide) constituent les spéculations les plus courantes et les 
plus couramment cultivées dans le milieu d'étude. Ces différentes plantes sont non seulement consommatrices de l'espace, mais contribuent à l'appauvrissement, à la dégradation des sols et donc de l'environnement. Les

\section{REFERENCES BIBLIOGRAPHIQUES}

ABE, 1999. Loi-cadre sur l'environnement en République du Bénin. Loi N98-030 du 12 Février 1999, portant Loi-cadre sur l'environnement en République du Bénin, ABE/MEHU, République du Bénin, Cotonou, $64 \mathrm{p}$.

Adewi E., 2012. Les stratégies agricoles de gestion de la péjoration pluviométrique au Togo. Thèse de Doctorat unique de géographie, UL, Lomé, $320 \mathrm{p}$.

Akissoé N., Hounhouigan D.J., Bricas N., Vernier P., Nago M.C. \& Olorunda O.A., 2001. Physical, chemical and sensory evaluation of dried yam (Dioscorea rotundata) tubers, flour and amala, a flour drived product. Tropical science, 41 (3) 151-155

Arouna 0., 2005. Carte phytoécologique de la forêt classée de Wari-Maro au Bénin. Mémoire de DEA/GEN/EDP/FLASH/UAC, Bénin, 99 p.

Arouna O., 2012. Cartographie et modélisation prédictive des changements spatio-temporels de la végétation dans la Commune de Djidja au Bénin : implications pour l'aménagement du territoire. Thèse de doctorat, Université d'Abomey-Calavi, Bénin, $246 p$.

Azontondé H. A., Feller C., Ganry F. \& Remy J. C., 1998. Le Mucuna et la restauration des propriétés d'un sol ferralitique au sud du Bénin. Agriculture et Développement $n^{\circ} 18$, CIRAD- Montpellier, France, 14-21.

Azontondé H.A., 2000. Dynamique de la matière organique et de l'azote dans le système Mucuna-maïs sur un sol ferralitique (terre de barre) au Sud-Bénin. Thèse de doctorat. ENSA-Montpellier, France, $241 \mathrm{p}$.

Boli Z., Roose E., BepAziem B., Sanon K. \& Waechter F., 1993. Effets des techniques culturales sur le ruissellement, l'érosion et la production de coton et maïs sur un sol ferrugineux tropical sableux du Nord Cameroun (M'bissiri, 1991 1992), Cah OrstomSérPédol ; 28 : 309-26.

CeRPA-Atacora/Donga, 2004. Rapport annuel d'activités : Campagne agricole 2003-2004. Natitingou, Bénin, $106 \mathrm{p}$. pratiques culturales inappropriées dégradent les sols et les systèmes de culture sans amendement réduisent considérablement les teneurs en matière organique et en azote.

CeRPA-Atacora/Donga, 2005. Rapport annuel d'activités : Campagne agricole 2004-2005. Natitingou, Bénin, $145 \mathrm{p}$.

CeRPA- Atacora/Donga, 2013. Rapport annuel d'activités : Campagne agricole 2012-2013. Natitingou, Bénin, $133 p$.

Démon A., 1991. Activités humaines et dégradation de l'environnement dans la Circonscription Urbaine de Kandi. Mémoire de Maîtrise de Géographie, FLASH-UNB, 101 p.

Dixon J., Gulliver A. et Gibbon D., 2001. Farming systems and poverty: improving farmers livelihoods in a changing word. Rome, Washington, FAO et Banque Mondiale.

FAO, 2003. Gestion de la fertilité des sols pour la sécurité alimentaire en Afrique subsaharienne. Rome, Italie, 63 p. En ligne: ftp://ftp.fao.org/docrep/fao/006/x9681f00.pdf, consulté le 15/04/13.

Honlonkou N. A., 1999. Impact économique des techniques de fertilisation des sols : cas de la jachère Mucuna au sud du Bénin. Thèse de Doctorat de 3è cycle. Côte d'ivoire : CIRES, $187 p$

ISRIC., 2007. Programme des Nations Unies sur l'évaluation scientifique sur l'état de la dégradation des sols causée par les activités humaines (GLASOD) $9 \mathrm{p}$.

Kiansi Y., 2011. Cogestion de la Réserve de Biosphère de la Pendjari : Approche concertée pour la conservation de la biodiversité et le développement économique local. Thèse de Doctorat Unique, Université d'Abomey-Calavi, 275p.

Kombienou P.D., 2010. Systèmes agricoles et conséquences des pratiques culturales sur les terres de la chaîne de l'Atacora : cas des terroirs de Boukombé. Mémoire de DEA, EDP/FLASH/UAC, Cotonou, Bénin, 107 p. + Annexes.

Kombienou P.D., Arouna O., Azontondé A. H., Mensah G. A. \& Sinsin B. A., 2015. Caractérisation du niveau de fertilité des sols de la chaîne de I'Atakora au nord-ouest du Bénin. Journal of Animal \& Plant Sciences, 25(2): 3836-3856. 
Kombienou P.D., Arouna O., Azontondé H. A., Mensah G. A. \& Sinsin A.B., 2016. Influences des activités agricoles sur la fertilité des sols de la chaîne de l'Atakora au nord-ouest du Bénin. Rev. Sc. Env. Univ., Lomé (Togo), 11(1) : 381404.

Kombienou P.D., 2016. Influences des systèmes de productions agricoles sur l'occupation des terres, la fertilité des sols et l'agro-biodiversité en zone montagneuse au nord-ouest du Bénin. Thèse de Doctorat Unique, EDP/FLASH/UAC, Cotonou, Bénin, 281 p. + Annexes.

Koundé C.Z., 1998. Etude de l'impact du compost à base d'ordures ménagères sur la fertilité d'un sol ferrugineux tropical à Djougou. Thèse d'ingénieur agronome. Cotonou, Bénin, FSA /UNB, $96 \mathrm{p}$.

Léopold L. B., Charke F. E., Hanshaw B. B. \& Bashley J. R., 1971. A procedure for evaluation environmental impacts. US Geological Survey Ciralar, Washington, D.C, 645 P.

Mulder I., 2000. Soil degradation in Benin: Farmers perception and responses. Tinbergen Institute, Researsh series $\mathrm{N}^{\circ} 240$ Vrije Universiteit Amsterdam, Hollande, $241 \mathrm{p}$.

Natta J. N. K., 1999. Tradition et développement: Occupation, exploitation du sol et organisation spatiale chez les Bètammaribè du Nord-Bénin. Mémoire de Maîtrise, UNB/Bénin, 1999, Cotonou, Bénin, $138 \mathrm{p}$.

Ouorou Barré F.I., 2014. Contraintes climatiques, pédologiques et production agricole dans l'Atacora (nord-ouest du Bénin).Thèse de Doctorat Unique, EDP/FLASH/UAC, 264 p.

Ramade F., 1991: http://msncoa.org./.../MSNCOA, 23rd Annual Conference Ramade Hilltop a NatchezConvention-Center-March7-10rd 1991 pdf.

Roufaï M. Chakirou, 2005. L'évaluation environnementale face aux enjeux d'un développement agricole durable en Afrique de l'Ouest.10ème Colloque international des spécialistes francophones en évaluation d'impacts. Angers du 20 au 24 Juin 2005. Doctorant à l'Ecole doctorale de ParisSorbonne UFR de Géographie et Aménagement.191, rue Saint Jacques 75005 Paris, France, 9p.

Zinzindohoué, 2012. Etat des lieux de la sécurité alimentaire dans le département de l'Atacora (au Nord-Ouest du Bénin) et analyse des politiques publiques. Master of Advanced studies en Action Humanitaire. CERAH GENEVE, 50p 